\title{
Geometry of Geometrically Finite One-Dimensional Maps
}

\author{
Yunping Jiang \\ Department of Mathematics, Queens College of CUNY, 65-30 Kissena Blvd, Flushing, \\ NY 11367, USA
}

Received: 1 October 1992/in revised form: 25 March 1993

\begin{abstract}
We study the geometry of certain one-dimensional maps as dynamical systems. We prove the property of bounded and bounded nearby geometry of certain $C^{1+\alpha}$ one-dimensional maps with finitely many critical points. This property enables us to give the quasisymmetric classification of geometrically finite one-dimensional maps.
\end{abstract}

\section{Contents}

1. Introduction . . . . . . . . . . . . . . . . . . . . . . . . . . . . . . . 639

2. Geometrically Finite One-Dimensional Maps . . . . . . . . . . . . . . . . . . 640

3. Estimates of Nonlinearity . . . . . . . . . . . . . . . . . . . . . . . . . . . . . . . . . 641

4. Bounded and Bounded Nearby Geometry . . . . . . . . . . . . . . . . . . . 644

5. Quasisymmetric Conjugacy . . . . . . . . . . . . . . . . . . . . . . . . . 645

References .. . . . . . . . . . . . . . . . . . . . . . . . 647

\section{Introduction}

Two smooth maps $f$ and $g$ from a one-dimensional $C^{2}$-Riemannian manifold $M$ into itself are topologically conjugate if there is a homeomorphism $h$ from $M$ onto itself such that $f \circ h=h \circ g$. A nontrivial problem [10] asked by Sullivan was about whether the conjugating map $h$ is necessarily quasisymmetric [1]. (We note that when $f$ and $g$ are both holomorphic and expanding maps on a domain in the Riemann sphere, then $h$ is quasiconformal [1] because of bounded geometry property $[9,10]$.) In [4 and 5], we studied this kind of problem for an interval map with one critical point. In this paper, we generalize the results of [4 and 5] to geometrically finite maps which are certain one-dimensional maps with finitely many critical points (see Definition 3 in Sect. 2). We prove that the induced sequence of nested partitions of $M$ by a geometrically finite map (see Sect. 2 for the definition) has bounded and bounded nearby geometry (see Definition 2 in Sect. 2). 
Theorem A. Suppose $f$ from $M$ into itself is geometrically finite and $\eta=\left\{\eta_{n}\right\}_{n=1}^{\infty}$ is the induced sequence of nested partitions of $M$ by $f$. Then $\eta$ is of bounded and bounded nearby geometry.

A homeomorphism $h$ from $M$ onto itself is quasisymmetric [1] if there is a constant $K>0$ so that for any two points $x$ and $y$ in $M$,

$$
K^{-1} \leq \frac{|h(x)-h(z)|}{|h(z)-h(y)|} \leq K
$$

where $z=(x+y) / 2$ is the midpoint of $x$ and $y$. Two maps $f$ and $g$ are quasisymmetrically conjugate if they are topologically conjugate and the conjugacy is quasisymmetric. From Milnor and Thurston's paper [8], any topological class of geometrically finite maps is determined by its kneading invariant. Using bounded and bounded nearby geometry, we can further prove that the quasisymmetric classes of geometrically finite maps are determined by their kneading invariants.

Theorem B. Suppose $f$ and $g$ from $M$ into itself are geometrically finite and topologically conjugate. They are then quasisymmetrically conjugate.

Before proving these theorems we will prove two important lemmas in Sect. 3 to estimate the nonlinearity of the iterates of a geometrically finite map. The reader may refer to [6] for a more general version of Lemma 2 in Sect. 3.

\section{Geometrically Finite One-Dimensional Maps}

Suppose $M$ is the interval $[-1,1]$ or the unit circle $S^{1}$ and $f$ from $M$ into itself is a $C^{1}$ map. A point $c \in M$ is said to be critical if $f^{\prime}(c)=0$ and it is said to have power law type at $c$ if there is a number $\gamma>1$ such that

$$
\lim _{x \mapsto c+} \frac{f^{\prime}(x)}{|x-c|^{\gamma-1}} \text { and } \lim _{x \mapsto c-} \frac{f^{\prime}(x)}{|x-c|^{\gamma-1}}
$$

have nonzero limits $A$ and $B$. Here $\gamma$ and $\tau=A / B$ are called the exponent and asymmetry of $f$ at $c$ [5]. Let $C=\left\{c_{1}, c_{2}, \ldots, c_{l}\right\}$ be the set of critical points of $f$. Henceforth, we will assume that all the critical points of $f$ are of power law type and $\Gamma=\left\{\gamma_{1}, \gamma_{2}, \ldots, \gamma_{l}\right\}$ is the set of corresponding exponents. Furthermore, we assume that $f$ maps the boundary of $M$ (if it is not empty) into itself and the one-sided derivative of $f$ at every boundary point of $M$ is nonzero.

Definition 1. We say that $f$ is $C^{1+\alpha}$ for some $0<\alpha \leq 1$ if

(*) the derivative $f^{\prime}$ of $f$ is $\alpha$-Hölder continuous, and

(**) for every critical point $c_{i}$ of $f$, there is a small neighborhood $U_{i}$ of $c_{i}$ in $M$ so that $r(x)=f^{\prime}(x) /\left|x-c_{i}\right|^{\gamma_{i}-1}$ is $\alpha$-Hölder on $\left\{x<c_{\imath}\right\} \cap U_{i}$ and on $\left\{x>c_{i}\right\} \cap U_{i}$.

Suppose the set of critical orbits $C O=\bigcup_{i=0}^{\infty} f^{\circ \imath}(C)$ is finite. Then $\eta_{1}=$ $\left\{L_{1}, \ldots, L_{d}\right\}$, the closures of the intervals in the complement of $C O$ in $M$, is a Markov partition ( namely $f\left(L_{\imath}\right)=\bigcup_{i_{k}} L_{\imath_{k}}$ for every $L_{i} \in \eta_{1}$ ). Let $\eta_{n}=\left\{I \mid f^{\circ n}(I)=\right.$ $L_{i}$ for some $L_{i}$ and $f^{\circ n} \mid I$ is a homeomorphism $\}$. We call $\eta_{n}$ the $n^{\text {th }}$-partition and $\eta=\left\{\eta_{n}\right\}_{n=1}^{\infty}$ the induced sequence of nested partitions of $M$ by $f$.

Definition 2. The induced sequence $\eta=\left\{\eta_{n}\right\}_{n=1}^{\infty}$ is said to be of bounded geometry if there is a constant $K>0$ so that the ratio $|J| /|I| \geq K$ for every pair $J \subset I$ with 
$J \in \eta_{n+1}$ and $I \in \eta_{n}$. And it is said to be of bounded nearby geometry if there is a constant $K>0$ so that the ratio $\left|J_{1}\right| /\left|J_{2}\right| \geq K$ for every pair $J_{1}$ and $J_{2}$ in $\eta_{n}$ with a common endpoint.

Remark 1 . We use $B_{f}$ to denote the largest possible value of $K$ in this definition.

Let $\lambda_{n}$ be the maximum of lengths of the intervals in $\eta_{n}$. Then $\eta_{n}$ is said to tend to zero exponentially if there are constants $K>0$ and $0<\mu<1$ such that $\lambda_{n} \leq K \mu^{n}$ for all positive integers $n$.

Definition 3. We say that $f$ is geometrically finite if

(1) $f$ is $C^{1+\alpha}$ for some $0<\alpha \leq 1$,

(2) $C O=\bigcup_{i=0}^{\infty} f^{\circ \imath}(C)$ is finite,

(3) no critical point is a periodic point of $f$,

(4) $\eta_{n}$ tends to zero exponentially.

Remark 2. (2) and (3) are equivalent to the statement that $f$ has only finitely many critical points and every critical point is preperiodic.

\section{Estimates of Nonlinearity}

We need the naive distortion lemma for one-dimensional maps.

The Naive Distortion Lemma. Suppose $g$ from $V$ into $M$ is a $C^{1+\alpha}$ map for some $0<\alpha \leq 1$ and $a_{0}=\inf _{x \in V}\left|g^{\prime}(x)\right|>0$. Let $b_{0}=\sup _{x \neq y \in V} \frac{\left|g^{\prime}(x)-g^{\prime}(y)\right|}{|x-y|^{\alpha}}<\infty$. Then for any two sequences $\left\{x_{\imath}\right\}_{\imath=1}^{n}$ and $\left\{y_{i}\right\}_{i=1}^{n}$ in $V$,

$$
\log \left(\prod_{\imath=1}^{n}\left|\frac{g^{\prime}\left(x_{\imath}\right)}{g^{\prime}\left(y_{i}\right)}\right|\right) \leq \frac{b_{0}}{a_{0}} \sum_{i=1}^{n}\left|x_{\imath}-y_{\imath}\right|^{\alpha} .
$$

Proof. The proof of this lemma is easy for

$$
\begin{aligned}
\log \left(\prod_{\imath=1}^{n}\left|\frac{g^{\prime}\left(x_{\imath}\right)}{g^{\prime}\left(y_{i}\right)}\right|\right) & \leq \sum_{i=1}^{n}|\log | g^{\prime}\left(x_{i}\right)|-\log | g^{\prime}\left(y_{\imath}\right)|| \\
& \leq \sum_{i=1}^{n} \frac{1}{a_{0}}\left|g^{\prime}\left(x_{i}\right)-g^{\prime}\left(y_{i}\right)\right| \leq \sum_{i=1}^{n} \frac{b_{0}}{a_{0}}\left|x_{\imath}-y_{\imath}\right|^{\alpha}
\end{aligned}
$$

Suppose $f$ from $M$ into itself is geometrically finite., Next two lemmas provide estimates for the nonlinearity of the iterates of $f$.

Let $U=\bigcup_{i=1}^{l} U_{i}$ be the union of $U_{i}$ in $(* *)$ of Definition 1 and $V=\overline{M \backslash U}$ be the closures of the complement of $U$ in $M$. By (4) of Definition 3, we can take $U_{i}$ so that every $U_{\imath}$ consists of two intervals in $\eta_{n_{0}}$ (for a fixed integer $n_{0}$ ) and, without loss of generality, we may assume that $\bar{U} \cap\left(\bigcup_{\imath=1}^{\infty} f^{\circ \imath}(C)\right)=\emptyset$.

Lemma 1. There is a constant $K>0$ such that if $f^{\circ i}(y)$ and $f^{\circ \imath}(x)$ are in the same connected component of $V$ for every $i=0,1, \ldots, n-1$, then

$$
\log \left(\frac{\left|\left(f^{\circ n}\right)^{\prime}(x)\right|}{\left|\left(f^{\circ n}\right)^{\prime}(y)\right|}\right) \leq K .
$$


Proof. It follows directly from the naive distortion lemma.

For the fixed integer $n_{0}$ and $x$ and $y$ in an interval $\tilde{I}$ in $\eta_{n_{0}}$, we use $I_{x y} \subset \tilde{I}$ to denote the interval bounded by $x$ and $y$.

Lemma 2. There is a constant $K>0$ so that if $f$ from $I_{x y}$ to $f^{\circ n}\left(I_{x y}\right)$ is injective and $f^{\circ n}\left(I_{x y}\right)$ is a subinterval of some $U_{i}$, then

$$
\log \left(\frac{\left|\left(f^{\circ n}\right)^{\prime}(x)\right|}{\left|\left(f^{\circ n}\right)^{\prime}(y)\right|}\right) \leq K .
$$

Remark 3. A more general version of this lemma appears in [6].

Proof. The ratio $\left|\left(f^{\circ n}\right)^{\prime}(x)\right| /\left|\left(f^{\circ n}\right)^{\prime}(y)\right|$ equals the product $\prod_{i=0}^{n-1}\left|f\left(x_{\imath}\right)\right| /\left|f^{\prime}\left(y_{i}\right)\right|$, where $x_{i}=f^{\circ i}(x)$ and $y_{\imath}=f^{\circ i}(y)$. We divide this product into two products,

$$
\prod_{x_{i}, y_{\imath} \in V} \frac{\left|f^{\prime}\left(x_{\imath}\right)\right|}{\left|f^{\prime}\left(y_{i}\right)\right|} \text { and } \prod_{x_{\imath}, y_{\imath} \in U} \frac{\left|f^{\prime}\left(x_{\imath}\right)\right|}{\left|f^{\prime}\left(y_{\imath}\right)\right|} \text {. }
$$

From the naive distortion lemma, there is a constant $K_{1}>0$ so that

$$
\log \left(\prod_{x_{\imath}, y_{i} \in V} \frac{\left|f^{\prime}\left(x_{\imath}\right)\right|}{\left|f^{\prime}\left(y_{i}\right)\right|}\right) \leq K_{1} .
$$

To estimate the second one, we write $\prod_{x_{i}, y_{\imath} \in U}\left|f^{\prime}\left(x_{\imath}\right)\right| /\left|f^{\prime}\left(y_{i}\right)\right|=I \cdot I I \cdot I I I$, where

$$
\begin{gathered}
I=\prod_{x_{\imath}, y_{\imath} \in U}\left(\frac{\left|x_{\imath}-c_{k_{\imath}}\right|^{\gamma_{k_{\imath}}}}{\left|f\left(x_{\imath}\right)-f\left(c_{k_{\imath}}\right)\right|} \frac{\left|f\left(y_{i}\right)-f\left(c_{k_{i}}\right)\right|}{\left|y_{\imath}-c_{k_{\imath}}\right|^{\gamma_{k_{\imath}}}}\right)^{m_{k_{\imath}}}, \\
I I=\prod_{x_{\imath}, y_{\imath} \in U}\left(\frac{\left|y_{\imath}-c_{k_{\imath}}\right|^{\gamma_{k_{\imath}}-1}}{\left|f^{\prime}\left(y_{i}\right)\right|} \frac{\left|f^{\prime}\left(x_{\imath}\right)\right|}{\left|x_{i}-c_{k_{\imath}}\right|^{\gamma_{k_{\imath}}-1}}\right),
\end{gathered}
$$

and

$$
I I I=\prod_{x_{\imath}, y_{i} \in U}\left(\frac{\left|f\left(x_{\imath}\right)-f\left(c_{k_{i}}\right)\right|^{m_{k_{\imath}}}}{\left|f\left(y_{\imath}\right)-f\left(c_{k_{\imath}}\right)\right|^{m_{k_{\imath}}}}\right),
$$

where $m_{k}=\left(\gamma_{k_{\imath}}-1\right) / \gamma_{k_{i}}$ if $x_{i}$ and $y_{i}$ are in $U_{k_{i}}$. If we take

$$
g_{i}^{\prime}(x)=\frac{\left|x-c_{k_{\imath}}\right|^{\gamma_{k_{\imath}}}}{\left|f(x)-f\left(c_{k_{\imath}}\right)\right|} \text { or } \frac{\left|x-c_{k_{\imath}}\right|^{\gamma_{k_{\imath}}-1}}{f^{\prime}(x)},
$$

then from $(* *)$ of Definition 1 and the naive distortion lemma, there is a constant $K_{2}>0$ such that

$$
\log (I \cdot I I) \leq K_{2}
$$

Now let us concentrate on the estimate of

$$
\log \left(\prod_{x_{\imath}, y_{\imath} \in U}\left(\frac{\left|f\left(x_{i}\right)-f\left(c_{k_{i}}\right)\right|}{\left|f\left(y_{i}\right)-f\left(c_{k_{\imath}}\right)\right|}\right)^{m_{k_{\imath}}}\right) .
$$


Write

$$
\frac{f\left(x_{\imath}\right)-f\left(c_{k_{\imath}}\right)}{f\left(y_{\imath}\right)-f\left(c_{k_{\imath}}\right)}=1+\frac{f\left(x_{\imath}\right)-f\left(y_{i}\right)}{f\left(y_{\imath}\right)-f\left(c_{k_{\imath}}\right)}
$$

then

$$
\begin{aligned}
\log \left(\prod_{x_{\imath}, y_{\imath} \in U}\left(\frac{\left|f\left(x_{\imath}\right)-f\left(c_{k_{\imath}}\right)\right|}{\left|f\left(x_{\imath}\right)-f\left(c_{k_{\imath}}\right)\right|}\right)^{m_{k_{\imath}}}\right) & \leq \sum_{s=1}^{r-1} \frac{1}{m_{k_{\imath_{s}}}} \log \left(1+\frac{\left|f\left(x_{i_{s}}\right)-f\left(y_{\imath_{s}}\right)\right|}{\left|f\left(x_{\imath_{s}}\right)-f\left(c_{k_{\iota_{s}}}\right)\right|}\right) \\
& \leq K_{3} \sum_{s=1}^{r-1} \frac{\left|f\left(x_{\imath_{s}}\right)-f\left(y_{\imath_{s}}\right)\right|}{\left|f\left(x_{\imath_{s}}\right)-f\left(c_{k_{\iota_{s}}}\right)\right|}
\end{aligned}
$$

where $i_{1}<i_{2}<\ldots<i_{r-1}<n$ and $K_{3}>0$ is a constant. Let $i_{r}=n$. Using Lemma 1, there is a constant $K_{4}>0$ such that for $0 \leq s<r$,

$$
\frac{\left|f\left(x_{\imath_{s}}\right)-f\left(y_{\imath_{s}}\right)\right|}{\left|f\left(x_{\imath_{s}}\right)-f\left(c_{k_{\imath_{s}}}\right)\right|} \leq K_{4} \frac{\left|x_{\imath_{s+1}}-y_{\imath_{s+1}}\right|}{\left|y_{\imath_{s+1}}-f^{\circ\left(\imath_{s+1}-\imath_{s}\right)}\left(c_{k_{\imath_{s}}}\right)\right|}
$$

Suppose $D>0$ is the distance between $U$ and the post-critical orbits $\bigcup_{\imath=1}^{\infty} f^{\circ \imath}(C)$. For $0 \leq s<r-1$, since $y_{\imath_{s+1}}$ is in $U$,

$$
\frac{\left|x_{\imath_{s+1}}-y_{\imath_{s+1}}\right|}{\left|y_{\imath_{s+1}}-f^{\circ\left(\imath_{s+1}-\imath_{s}\right)}\left(c_{k_{\imath_{s}}}\right)\right|} \leq K_{4} \frac{\left|x_{\imath_{s+1}}-y_{\imath_{s+1}}\right|}{D} \text {. }
$$

For $s=r-1$, by the hypothesis, $y_{\imath_{r}}$ is in $U$ too. So

$$
\frac{\left|x_{\iota_{r}}-y_{\imath_{r}}\right|}{\left|y_{\imath_{r}}-f^{\circ\left(\imath_{r}-\imath_{s}\right)}\left(c_{k_{\imath_{r-1}}}\right)\right|} \leq K_{4} \frac{\left|x_{\imath_{r}}-y_{\imath_{r}}\right|}{D} .
$$

Hence there is a constant $K_{5}>0$ such that

$$
\log \left(\prod_{x_{\imath}, y_{\imath} \in U} \frac{\left|f\left(x_{\imath}\right)-f\left(c_{k_{\imath}}\right)\right|^{m_{k_{\imath}}}}{\left|f\left(x_{\imath}\right)-f\left(c_{k_{\imath}}\right)\right|^{m_{k_{\imath}}}}\right) \leq K_{5} .
$$

Combining all the estimates together we get a constant $K>0$ satisfying the lemma.

Remark 4. From the proof of Lemma 2, one can see that the distortion of $f$ along an orbit is controlled by $\left|x_{n}-y_{n}\right| / \operatorname{dist}\left(y_{n}, \bigcup_{l=1}^{\infty} f^{\circ \imath}(C)\right)$ even if the orbit may visit the neighborhood $U$ of the set $C$ of critical points of $f$ many times where dist means the distance. The reader may compare this with the Koebe distortion property in one complex variable [2]. 


\section{Bounded and Bounded Nearby Geometry}

We prove that the sequence $\left\{\eta_{n}\right\}_{n=1}^{\infty}$ of nested partitions of $M$ by a geometrically finite map $f$ has bounded and bounded nearby geometry in this section.

Theorem A. Suppose $f$ from $M$ into itself is geometrically finite and $\eta=\left\{\eta_{n}\right\}_{n=1}^{\infty}$ is the induced sequence of nested partitions of $M$ by $f$. Then $\eta$ is of bounded and bounded nearby geometry.

Proof. Let $n_{0}$ be the fixed integer in Sect. 3 (before Lemma 1) and $K_{1}>0$ be the minimum of ratios $|J| /|I|$ for $J \subset I$ with $J \in \eta_{j+1}$ and $I \in \eta_{j}$ for $1 \leq j \leq n_{0}$.

For a pair $J \subset I$ with $J \in \eta_{k+1}$ and $I \in \eta_{k}$ and $n=k-n_{0}>0$, let $J_{i}=f^{\circ i}(J)$ and $I_{\imath}=f^{\circ i}(I)$ for $i=0, \ldots, n$. Then $J_{n} \in \eta_{n_{0}+1}$ and $I_{n} \in \eta_{n_{0}}$. We consider the intervals $\left\{I_{0}, \ldots, I_{n}\right\}$ in two cases: (i) no one of them is in $U$ and (ii) at least one of them is in $U$.

In the case (i), applying Lemma 1 , there is a constant $K_{2}>0$, such that

$$
\frac{\left|\left(f^{\circ n}\right)^{\prime}(y)\right|}{\left|\left(f^{\circ n}\right)^{\prime}(x)\right|} \geq K_{2}
$$

for $x$ and $y$ in $I$. This implies that

$$
\frac{|J|}{|I|} \geq K_{3}=K_{2} K_{1}
$$

In the case (ii), let $l \leq n$ be the greatest integer so that $I_{l} \subset U$. We note that $I_{\imath} \subset V$ for $i=l+1, \ldots, n$. Applying Lemma 1 again as in the case (i), we have that

$$
\frac{\left|J_{l+1}\right|}{\left|I_{l+1}\right|} \geq K_{3} \text {. }
$$

Suppose $I_{l}$ is contained in $U_{i}$. Because $f \mid U_{i}$ is comparable with the map $q_{\imath}(x)=\left|x-c_{\imath}\right|^{\gamma_{i}}+f\left(c_{\imath}\right)$, there is a constant $K_{4}>0$ (only depends on $K_{3}$ ) so that

$$
\frac{\left|J_{l}\right|}{\left|I_{l}\right|} \geq K_{4} .
$$

Now applying Lemma 2, we have a constant $K_{5}>0$ so that

$$
\frac{\left|\left(f^{\circ(n-l)}\right)^{\prime}(y)\right|}{\left|\left(f^{\circ(n-l)}\right)^{\prime}(x)\right|} \geq K_{5}
$$

for $x$ and $y$ in $I$. This implies that

$$
\frac{|J|}{|I|} \geq K_{6}=K_{5} K_{4} .
$$

Hence $\eta$ is of bounded geometry.

To prove $\eta$ is of bounded nearby geometry, let $n_{1}>n_{0}$ be an integer such that if a pair $J_{1}$ and $J_{2}$ in $\eta_{n_{1}}$ with a common endpoint then either both of them are in $U$ or no endpoints of $J_{1}$ and $J_{2}$ are critical points of $f$. Suppose $K_{7}>0$ is the minimum of ratios $\left|J_{1}\right| /\left|J_{2}\right|$ for $J_{1}$ and $J_{2}$ with a common endpoint for $1 \leq j \leq n_{1}$.

Now for $k \geq n_{1}$ and $J_{1}$ and $J_{2}$ with a common endpoint, let $J_{1, i}=f^{\circ 2}\left(J_{1}\right)$ and $J_{1, \imath}=f^{\circ i}\left(J_{2}\right)$ for $i=0, \ldots, n=k-n_{1}$. We consider $\left\{J_{1, \imath}\right\}_{\imath=0}^{n}$ and $\left\{J_{2, i}\right\}_{\imath=0}^{n}$ in two 
cases: (a) for some $0<l \leq n, J_{1, l}=J_{2, l}$ and (b) $J_{1, i}$ and $J_{2, i}$ are all different for $i=0, \ldots, n$.

In (a), let $l$ be the smallest such integer, then the common endpoint of $J_{1, l-1}$ and $J_{2, l-1}$ is a critical point of $f$. It is easy to see that there is a constant $K_{8}>0$ such that

$$
\frac{\left|J_{1, l-1}\right|}{\left|J_{2, l-1}\right|} \geq K_{8} .
$$

Since $J_{1, l-1} \cup J_{2, l-1}$ is a subinterval of $U$, by applying Lemma 2, we have a constant $K_{9}>0$ such that

$$
\frac{\left|\left(f^{\circ(l-1)}\right)^{\prime}(y)\right|}{\left|\left(f^{\circ(l-1)}\right)^{\prime}(x)\right|} \geq K_{9}
$$

for $x$ and $y$ in $J_{1} \cup J_{2}$. This implies that

$$
\frac{\left|J_{1}\right|}{\left|J_{2}\right|} \geq K_{10}=K_{9} K_{8} .
$$

In (b), by using almost the same arguments as those in the proof of bounded geometry, we have a constant $K_{11}>0$ such that

$$
\frac{\left|J_{1}\right|}{\left|J_{2}\right|} \geq K_{11}
$$

Hence $\eta$ is of bounded nearby geometry. This completes the proof of Theorem A.

\section{Quasisymmetric Conjugacy}

Using bounded and bounded nearby geometry, we prove that any topological class of geometrically finite maps is actually a quasisymmetric class.

Theorem B. Suppose $f$ and $g$ from $M$ into itself are geometrically finite and topologically conjugate. They are then quasisymmetrically conjugate.

Remark 5. Some other interesting results about quasisymmetric classification have been proved in $[3,7$, and 11$]$.

Proof. Suppose $h$ is the conjugacy between $f$ and $g$, namely $h \circ f=g \circ h$, and $B_{f}$ and $B_{g}$ are the constants in Remark 1. Let $\eta_{f}=\left\{\eta_{n, g}\right\}_{n=1}^{+\infty}$ and $\eta_{g}=\left\{\eta_{n, g}\right\}_{n=1}^{+\infty}$ be the induced sequence of nested partitions of $M$ by $f$ and $g$ respectively.

For any $x<y$ in $M$, let $z=(x+y) / 2$ be the midpoint of $x$ and $y$ and $N>0$ be the smallest integer such that there is an interval $I$ in $\eta_{N, f}$ contained in $[x, y]$. Let $\tilde{I}$ be the interval in $\eta_{N-1, f}$ containing $I$. Then the union of $\tilde{I}$ and one of its adjacent 
intervals in $\eta_{N-1, f}$ contains $[x, y]$ (see Figs. 1-3). Because of bounded and bounded nearby geometry of $\eta_{g}$ (and refer to Fig. 1-3), there is a constant $K_{1}=K_{1}\left(B_{f}\right)>0$ such that

$$
\frac{|h(I)|}{|h([x, z])|} \geq K_{1} \quad \text { and } \quad \frac{|h(I)|}{|h([z, y])|} \geq K_{1} .
$$

Fig. 1

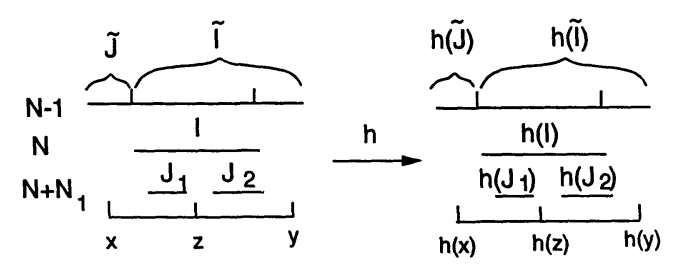

Fig. 2

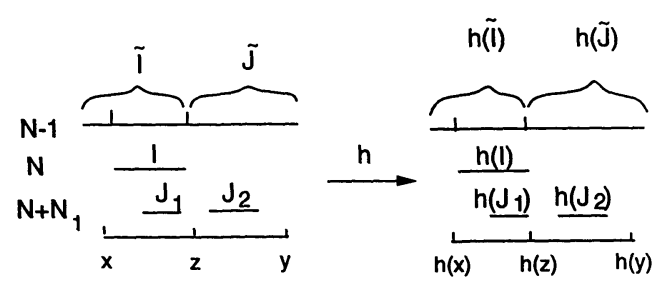

Fig. 3

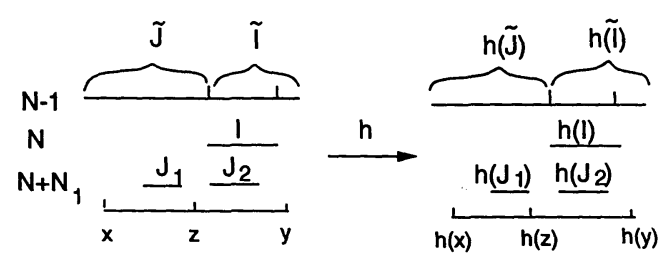

Because $\eta_{n, f}$ tends to zero exponentially and $\eta_{f}$ is of bounded and bounded nearby geometry, we can find a constant integer $N_{1}=N_{1}\left(B_{f}\right)>0$ such that there are intervals $J_{1}$ and $J_{2}$ in $\eta_{N+N_{1}}$ contained in $[x, z]$ and $[z, y]$, respectively. This implies that $h\left(J_{1}\right)$ and $h\left(J_{2}\right)$ are contained in $h([x, z])$ and $h([z, y])$ respectively. Because of bounded and bounded nearby geometry of $\eta_{g}$ again, there is a constant $K=K\left(N_{1}, B_{g}\right)>0$ (see Fig. 2-3) such that

$$
K^{-1} \leq \frac{|h(x)-h(z)|}{|h(z)-h(y)|} \leq K,
$$

which shows that $h$ is quasisymmetric.

Acknowledgements. The author would like to thank Prof. Dennis Sullivan for many suggestions. He would also like to thank the Institute for Mathematical Sciences at Stony Brook for its hospitality. 


\section{References}

1. Ahlfors, L.A.: Lectures on quasiformal maps. D. von Nostrand 1966

2. Bieberbach, L.: Conformal mapping. New York: Chelsea Publishing 1953

3. Cawley, E.: The Teichmüller space of an Anosov diffeomorphism of $T^{2}$. IMS preprint series 1991/8, SUNY at Stony Brook

4. Jiang, Y.: Leading gap determine the geometry of Cantor set. Preprint 1988, Graduate Center of CUNY and IHES preprint June 1989

5. Jiang, Y.: Generalized Ulam-von Neumann transformation. Thesis, 1990, Graduate Center of CUNY

6. Jiang, Y.: Dynamics of certain smooth one-dimensional maps. I. The $C^{1+\alpha}$-Denjoy-Koebe distortion lemma and II. Geometrically finite one-dimensional maps. IMS preprint series 1991/1, SUNY at Stony Brook

7. Jiang, Y.: On quasisymmetrical classification of infinitely renormalizable maps, I. Maps with the Feigenbaum's topology and II. Remarks on maps with a bounded type topology. IMS preprint series 1992/19, SUNY at Stony Brook

8. Milnor, J., Thurston, W.: On iterated maps of the interval I and II. Preprint, 1977, Princeton University

9. Sullivan, D.: Seminar on conformal and hyperbolic geometry. IHES preprint, March 1982

10. Sullivan, D.: Class notes, 1987

11. Sullivan, D.: Bounds, quadratic differentials, and renormalization conjectures. Providence, RI: American Mathematical Society Centennian Publications, Volume 2: Mathematics into the Twenty-first Century

Communicated by S.-T. Yau 
